Dieses Dokument ist eine Zweitveröffentlichung (Verlagsversion) /

This is a self-archiving document (published version)

„Dieser Beitrag ist mit Zustimmung des Rechteinhabers aufgrund einer (DFG-geförderten) Allianzbzw. Nationallizenz frei zugänglich."

This publication is openly accessible with the permission of the copyright owner. The permission is granted within a nationwide license, supported by the German Research Foundation (abbr. in German DFG).

https://www.nationallizenzen.de
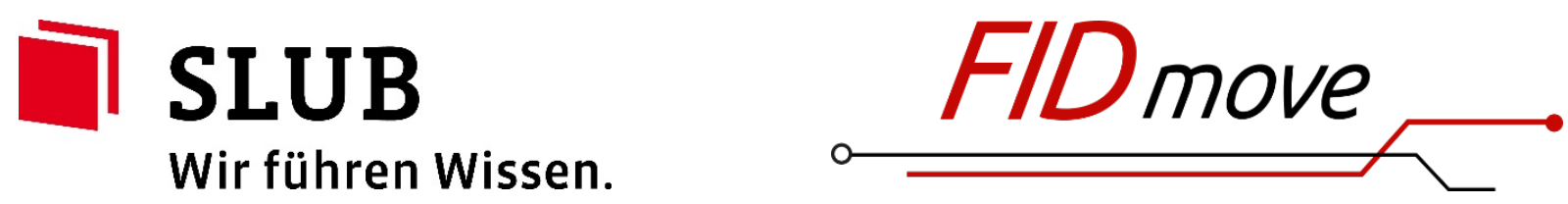


\title{
Multi-objective design of complex aircraft structures using evolutionary algorithms
}

\author{
J Seeger* and K Wolf \\ Institute of Aerospace Engineering, TU Dresden, Dresden, Germany
}

The manuscript was received on 2 November 2010 and was accepted after revision for publication on 4 May 2011.

DOI: $10.1177 / 0954410011411384$

\begin{abstract}
In this article, a design methodology for complex composite aircraft structures is presented. The developed approach combines a multi-objective optimization method and a parameterized simulation model using a design concept database. Due to the combination of discrete and continuous design variables describing the structures, evolutionary algorithms are used within the presented optimization approach. The approach requires an evaluation of the design alternatives that is performed by parameterized simulation models. The variability of these models is achieved using a design concept database that contains different layouts for each implemented structural part. Due to the complexity of the generated aircraft structures, the finite element method is applied for the calculation of the structural behaviour. The applicability of the developed design approach will be demonstrated by optimizing two composite aircraft fuselage examples. The obtained results show that the developed methodology is useful and reliable for designing complex aircraft structures.
\end{abstract}

Keywords: multi-objective design optimization, evolutionary algorithms, design concept database

\section{INTRODUCTION}

The outstanding weight-specific properties of carbon fibre reinforced plastics (CFRPs) make them well suited for applications in primary structural parts of aircrafts. Moreover, those materials provide the opportunity for tailored structural parts. Due to the layered structure and orthotropic material properties of each layer, they can be customized to the load and thus lead to minimum structural weight. Unfortunately, this ability results in a significant number of design variables that have to be optimized within the design process. It becomes more complex if the design concepts for the different structural parts have to be selected too. Moreover, additional objectives like deformation constraints or manufacturing costs have to be considered to develop capable

*Corresponding author: Institute of Aerospace Engineering, TU Dresden, 1062 Dresden, Germany.

email: joerg.seeger@tu-dresden.de structures. Therefore, the design of complex aircraft structures made of CFRPs becomes a challenging problem.

To solve this task efficiently, an automated design process is aspired. Therefore, a parameterized model is required that generates and simulates different design alternatives. Using the opportunities CFRP structures offer, this model needs the ability to create a wide spectrum of different structures. This spectrum should contain different design concepts like sandwich or monolithic skin concepts as well as different stiffener cross-section shapes. To handle these concepts and shapes in the parameterized model, they can be defined as discrete numbers. Describing the design alternatives, additional design variables are necessary as well. They represent the number of each part or their dimensions. Therefore, each structure is described by discrete and continuous numerical values. An efficient way to organize the parts and design concepts is the use of a database. This approach supports the further enhancement of the parameterized model by integration of new parts 
or design concepts. Based on the results of the simulation, evaluation criteria are determined that represent the structural fitness.

The information provided by the parameterized model can be used within an optimization approach to determine the optimal designs. To find the most efficient optimization approach, several requirements resulting from the model have to be considered. On the one hand, a flexible and robust optimization approach is needed, which is able to handle the appearing discrete and continuous design variables and solve combinatorial problems. On the other hand, the optimization approach needs the ability to consider multi-objective problems. Thus, an optimization approach based on evolutionary algorithms (EAs) was chosen for this study. The EAs have the ability to handle the combination of design variables due to their independence from derivative information of the objective function. They are used in several applications dealing with the optimization of CFRP structures. One group of algorithms within the EAs are the genetic algorithms (GAs). They are used in references [1] to [5] to find minimum-weight designs of stringer stiffened composite panels and in Gantovnik et al. [6] to optimize composite sandwich panels considering the same objective. Another application of GAs is presented in Liu et al. [7] and Soremekun et al. [8], where the algorithms are applied to find the optimal stacking sequence of laminate plates. The evolution strategies (ESs) are another kind of EAs. Applying this type of algorithm, the optimization of composite aircraft panels is presented in Kaletta and Wolf [9]. A third type of EA is the differential evolution (DE). In Spallino [10], a CFRP fuselage section is optimized using DE. The parallel application of several EAs was investigated in Wolf [11] to optimize sandwich panels for aircraft fuselages. A similar approach is presented in Keller [12]. Multiple EAs are combined and adopted for ply angle optimizations. The method is applied to find the minimum compliance design of an aircraft side ruder and a racing car rear wing design with the highest possible lowest natural frequency. The EAs allow the consideration of multiple objectives as well. In Spallino and Rizzo [13], a multi-objective approach using ESs is applied to optimize a laminated plate to find a solution with the smallest possible deformation as well as the minimal possible first natural frequency. Another application of the multiobjective optimization using EAs is presented in Irisarri et al. [14]. A laminated composite structure is optimized considering the objectives of minimum weight and minimum number of plies. In Seeger and Wolf [15], a parallel application of EAs is applied to find adaptive rotor blades with maximum deformation and minimum weight.

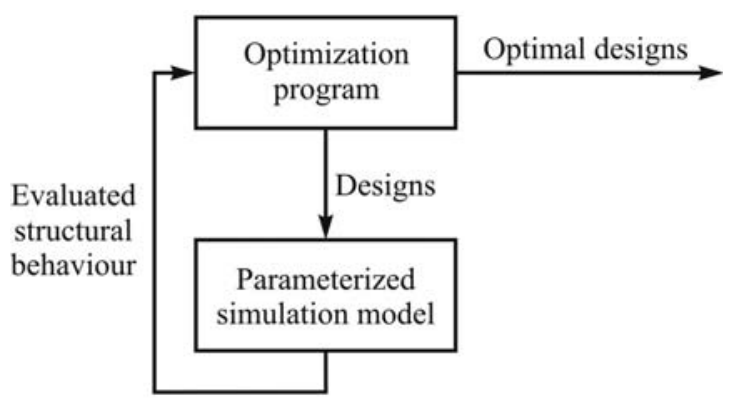

Fig. 1 Overview of the design methodology

The combination of the parameterized simulation model and the optimization approach using EAs creates a multi-objective design methodology (Fig. 1), which is presented in this article. The first section contains the implementation of the optimization approach in the optimization tool GEOpS ${ }^{2}$ (genetic and evolutionary optimization of structures), whereas in the following section, the general approach for the parameterized simulation model is presented. Finally, these fundamentals are applied to develop a parameterized simulation model for the multi-objective optimization of aircraft fuselage structures. To prove the applicability of the design methodology, the optimization tool GEOpS ${ }^{2}$ in combination with the developed model is used to find the optimal design solution for a composite test panel and a CFRP fuselage tail section.

\section{DESIGN METHODOLOGY}

\subsection{Optimization approach}

\subsubsection{Fundamentals}

Structural optimization involves the task of finding the best structural design, taking into account the given restrictions. In order to achieve this aim, an initial design solution has to be changed. The potential for change is expressed in terms of permissible ranges of a group of design variables which form the design vector $\boldsymbol{x}$

$$
\boldsymbol{x}=\left(x_{1}, x_{2}, \ldots, x_{n}\right)^{\mathrm{T}}
$$

The design variables $x_{i}$ are parameters which represent properties of the structure such as geometries or materials. Depending on the type of problem, design variables have to be expressed either as continuous or discrete values. All design variables constitute the design space $\Omega$

$$
\begin{aligned}
& x_{i} \in D_{i} \subset \Re^{1}, i=1(1) n \\
& \Omega:=D_{1} \times D_{2} \times \cdots \times D_{n} \subset \Re^{n} .
\end{aligned}
$$


The aim of the optimization process is to find among all feasible design vectors $\boldsymbol{x}$ the one that minimizes or maximizes an objective or fitness function

$$
\min f(\boldsymbol{x}) \text { and } \boldsymbol{x} \in \Omega
$$

The design space $\Omega$ is restricted by inequality and equality constraints

$$
\begin{aligned}
& \boldsymbol{g}(\boldsymbol{x}) \geq 0, \boldsymbol{g}(\boldsymbol{x})=\left(\boldsymbol{g}_{1}(\boldsymbol{x}), \boldsymbol{g}_{2}(\boldsymbol{x}), \ldots, \boldsymbol{g}_{m}(\boldsymbol{x})\right)^{\mathrm{T}}, \\
& \boldsymbol{g}: \Omega \rightarrow \mathfrak{R}^{m} \\
& \boldsymbol{h}(\boldsymbol{x})=0, \boldsymbol{h}(\boldsymbol{x})=\left(\boldsymbol{h}_{1}(\boldsymbol{x}), \boldsymbol{h}_{2}(\boldsymbol{x}), \ldots, \boldsymbol{h}_{l}(x)\right)^{\mathrm{T}}, \\
& \boldsymbol{h}: \Omega \rightarrow \mathfrak{R}^{l}
\end{aligned}
$$

In case of structural optimization problems, typical constraints are stress and strain allowables or deformation limits. The constrained optimization problem is given by

$$
\min f(\boldsymbol{x}) \text { and } \boldsymbol{x} \in \Omega, \boldsymbol{g}(\boldsymbol{x}) \geq 0, \boldsymbol{h}(\boldsymbol{x})=0
$$

Regarding the constraints, the whole design space $\Omega$ is divided into two disjoint sets, a feasible region $\Omega_{\mathrm{f}}$ and an infeasible one $\Omega_{\text {inf }}$

$$
\begin{aligned}
\Omega_{\mathrm{f}} & :=\{\boldsymbol{x} \in \Omega \mid \boldsymbol{g}(\boldsymbol{x}) \geq 0, \boldsymbol{h}(\boldsymbol{x})=0\} \subseteq \Omega \\
\Omega_{\mathrm{inf}} & :=\Omega \backslash \Omega_{\mathrm{f}}
\end{aligned}
$$

For the problem considered here, the constraints are not available in a closed form. Therefore, a penalty approach [16] is applied. A positive penalty term $P(\boldsymbol{g}(\boldsymbol{x}), \boldsymbol{h}(\boldsymbol{x}))$ is added to get the functional value of the alternative objective function $f^{E}(\boldsymbol{x})$, if constraints are violated

$$
f^{E}(\boldsymbol{x}):= \begin{cases}f(\boldsymbol{x}) & \forall \boldsymbol{x} \in \Omega_{f} \\ f(\boldsymbol{x})+P(\boldsymbol{g}(\boldsymbol{x}), \boldsymbol{h}(\boldsymbol{x})) & \forall \boldsymbol{x} \in \Omega_{\mathrm{inf}}\end{cases}
$$

Instead of the objective function $f(\boldsymbol{x})$, the alternative objective function $f^{E}(x)$ has to be minimized

$$
\min f^{E}(\boldsymbol{x}) \text { and } \boldsymbol{x} \in \Omega
$$

Up to this point, the description of the structural optimization procedure covers only the case of one objective function. However, in real-life problems, often conflicting objectives have to be considered within the optimization. Therefore, it might be difficult to combine them in one objective function. In this case, the multi-objective optimization offers a possibility to overcome this problem $[17,18]$.

For aircraft structures, the conflicting objectives might be the reduction of the mass, the deformation, or the manufacturing costs. Therefore, an arbitrary number, $q$, of alternative objective functions is defined which forms the alternative objective function vector:

$$
\boldsymbol{f}^{E}(\boldsymbol{x})=\left(f_{1}^{E}(\boldsymbol{x}), f_{2}^{E}(\boldsymbol{x}), \ldots, f_{q}^{E}(\boldsymbol{x})\right)^{\mathrm{T}}
$$

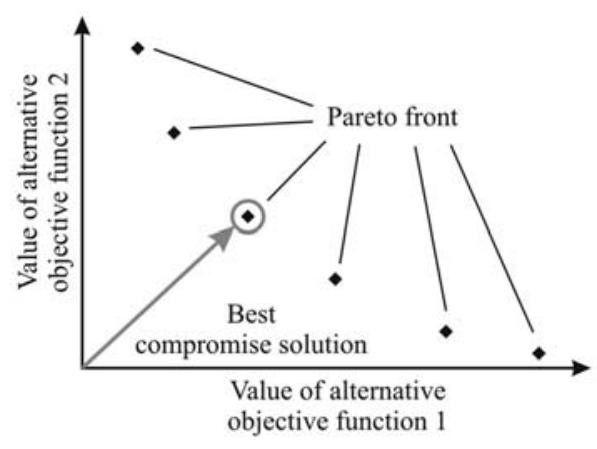

Fig. 2 Schematic of a Pareto front

This vector has to be minimized. Since it is rather unlikely that the same design vector $x$ will result in the best values for all alternative objective functions, it is necessary to use an approach that can handle different values. For this purpose, the Pareto optimality criterion is applied. It can be formulated as: A design $\boldsymbol{x}^{i}$ with the alternative objective function vector $\boldsymbol{f}^{E}\left(\boldsymbol{x}^{i}\right)$ is Pareto optimal, if there exists no other design $\mathbf{x} \in \Omega$ that dominates $\boldsymbol{x}^{\boldsymbol{i}}$. This means in case of $q$ alternative objective functions

$$
\forall \boldsymbol{x}, \boldsymbol{x} \neq \boldsymbol{x}^{i} \quad \exists n \in\{1,2, \ldots, q\} \quad \text { with } \quad f_{n}\left(\boldsymbol{x}^{i}\right) \leqslant f_{n}(\boldsymbol{x})
$$

Mostly, this approach does not provide a single solution, but rather a set of solutions called the Pareto optimal set. The plot of the alternative objective functions whose design vectors are in this Pareto optimal set is called the Pareto front (Fig. 2). The solution with the shortest position vector can be regarded as the best compromise solution.

\subsubsection{Evolutionary algorithms}

EAs mimic the principles of the biological evolution process. The optimization process starts with a population of different design solutions. Based on the information from these parent individuals, an offspring population is created using a number of evolutionary operators. The selection of better individuals leads to a progress in the optimization. The selection is based only on the computed values of the alternative objective function. Therefore, no derivative information is required. This makes it possible to find optimal solutions in discontinuous design spaces with combined discrete and continuous design variables. Although the basic principle is the same for all types of EAs, they differ considerably in the coding of the design variables and the way operators are working. In this research, GAs, ESs, and DE are employed. A short description of these EAs is given in the following paragraphs. 


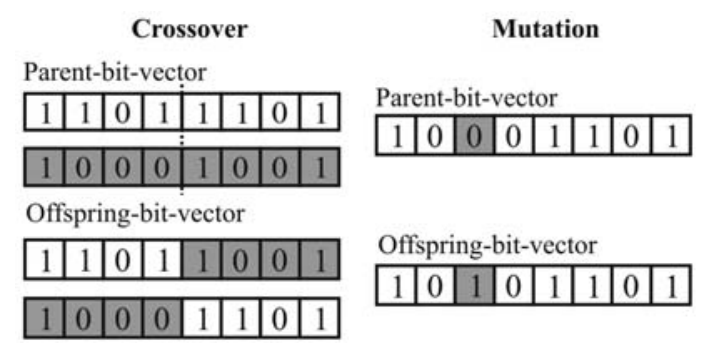

Fig. 3 Crossover and mutation operator of GAs

GAs are based on binary-coded design variables, which are combined in strings or chromosomes. These strings are modified by operators in order to find better solutions. Applying crossover, the main operator of the GAs, string sections of different individuals are changed between each other, as shown in the left part of Fig. 3. Another operator is the genetic mutation. This operator swaps single bits of the binary string (right part of Fig. 3). That means, smaller and bigger changes in the design variables depend on the location of the mutation. The newly generated design alternatives are considered in the following selection process in which the new parent population is formed. The creation of new individuals and the selection process alternate until a stop criterion terminates the optimization run. Due to the binarycoded parameters, this kind of operator is particularly well suited to discrete and combinatorial problems like the selection of design concepts as well as the placement of structural parts. A detailed description of the applied GAs can be found in Kaletta [19].

For the search in design spaces with combined continuous and discrete design variables, the application of ESs is more preferable. This method is presented in Bäck et al. [20] and is based on realvalued coding. The mutation is the most important operator of the ESs. It is based on a Gaussian probability function centred at the point of the original design variable. Based on this distribution, a step size is determined to create a new individual. Small step sizes are very common. Large ones are rare, but possible.

Another operator called recombination allows the exchange of design information between several individuals. Different types of the recombination operators are implemented in the optimization tool. In order to generate an offspring individual, single design variables of specific individuals are taken over or mean values are computed. For a better understanding, an example is given in Fig. 4, where the creation of two offspring individuals is presented. The shown individuals consist of three design variables that are modified by these two operators. Afterwards, a selection operation follows which works in the same way as with GAs.

The DE presented in Storn and Price [21] is the third type of EA that has been applied in this study. This method is closely related to the ESs. The DE is also based on real-coded design variables. The determination of the step size in the mutation process involves the computation of differential vectors between the design points of the parent individuals. An increasing homogeneity in the population causes a reduction of the step size and finally enforces a precise adjustment of the optimized individuals in the final phase of the optimization. Thus, the DE represents an intermediate state between the stochastic algorithm types such as GAs and ESs and purely deterministic algorithms. Also, DE uses selection operators which are different from the other two methods. The new parent population is formed by the comparison of each parent individual and its offspring. The DE is well suited for non-convex continuous problems and offers advantages for local search.

As described in the paragraphs before, each of the mentioned EAs has its special field of application. In order to combine the advantages of these methods,

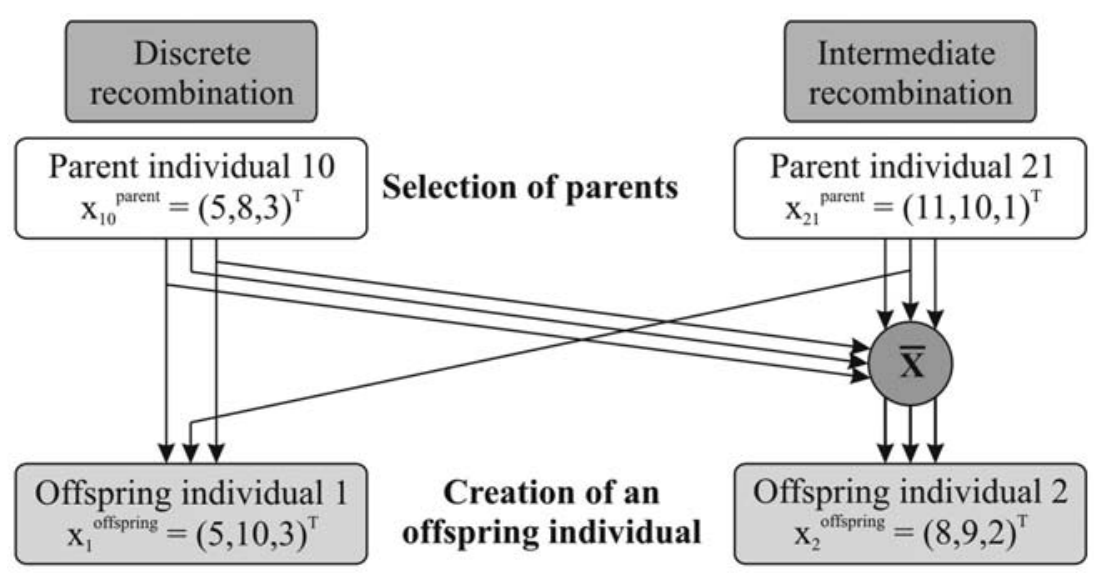

Fig. 4 Discrete and intermediate recombination 
all of them are implemented in the developed optimization tool named GEOpS ${ }^{2}$. It is possible but not always necessary to use all algorithms in parallel. Depending on the kind of problem, a single method can be also applied.

The used algorithms have proved their robustness and applicability for several optimization problems $[\mathbf{9}, \mathbf{1 1}, \mathbf{1 2}, \mathbf{1 5}]$. New EAs $[\mathbf{2 2}, \mathbf{2 3}]$ or other natureinspired metaheurisics $[\mathbf{2 4}, \mathbf{2 5}]$ are under investigation but will be implemented only if they achieve better results in benchmarks $[\mathbf{2 6}, \mathbf{2 7}]$ than the ones presented in this study.

\subsubsection{Efficiency-enhancing methods}

The application of EAs results in considerable computational effort. Due to the fact that no derivative information will be used, a lot of design variants have to be simulated and evaluated. Therefore, means are required to accelerate the optimization process. In Kaletta [19], different kinds of efficiencyenhancing methods were presented to optimize an aircraft fuselage panel with EAs. Two of them have been implemented in $\mathrm{GEOpS}^{2}$.

The inherent parallel structure of EAs makes them ideal candidates for parallelization. Since the algorithms work on the individuals of the population independently, it is straightforward to parallelize several aspects of the optimization procedure.

As mentioned before, it is necessary to evaluate the fitness of the individuals for an efficient selection. For complex structural optimization problems, a finite element analysis code is used, which requires a considerable computational effort. Due to the population-based approach of EAs, all newly created individuals are available at the same time. This permits to evaluate them in parallel. In GEOpS ${ }^{2}$, the message passing interface (MPI) is used to execute the parallel evaluation. MPI represents a standard for parallel computation in multi-processor environments. Therefore, PC clusters, PC with multi-core CPU as well as high-performance computers can be employed.
Another way to accelerate the optimization is the application of a technique called design space adjustment. In GEOpS ${ }^{2}$, a rather simple approach is used: the feasible region of the design space is reduced during the optimization run, depending on the parameter range of the best individuals (i.e. structural designs). An example for this method is given in Fig. 5. For the number of stiffeners of a stiffened panel, the initial design space has a range $0-5$. If the best individuals of the population have only two to three stiffeners after a number of iterations, it can be assumed that the best solutions will be in this interval. Therefore, the design space can be reduced. In order to prevent a premature stagnation of the optimization, a margin of safety is introduced for the new interval. In the given example, an additional stiffener is added on both sides, so that the new boundaries of the design space are one and four. Hence, in further iterations, only individuals with a number of stiffeners taken from this interval will be created.

\subsection{Parameterized simulation model}

The parameterized simulation model is the second part of the presented design methodology. As described before, it is needed to evaluate the designs for the selection process of the optimization. Due to the complexity of the models, the finite element method is used for simulation. To find the optimal solution among all feasible structural architectures, the model needs the ability to generate different design concepts of all parts. The approach presented here uses a design concept database to offer this flexibility. Within the database, the layouts of the parts are saved. Additionally, rules are implemented based on how the parts depend on each other. Thus, new architectures as well as further dependencies of the parts can be easily implemented.

To generate the model, several steps have to be performed. A flowchart of this process is depicted in Fig. 6. At the beginning, the optimization tool delivers a set of values for the design variables that represent the structural parts and their dimensions.

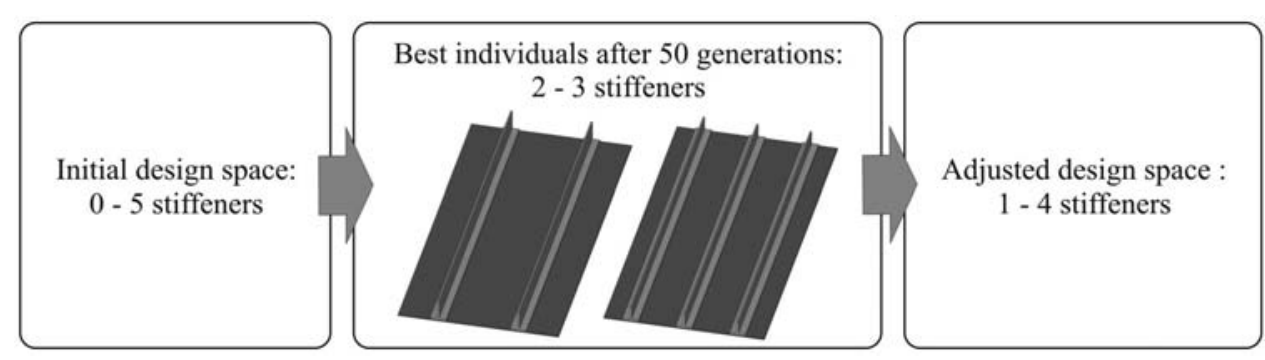

Fig. 5 Adjustment of the design space 


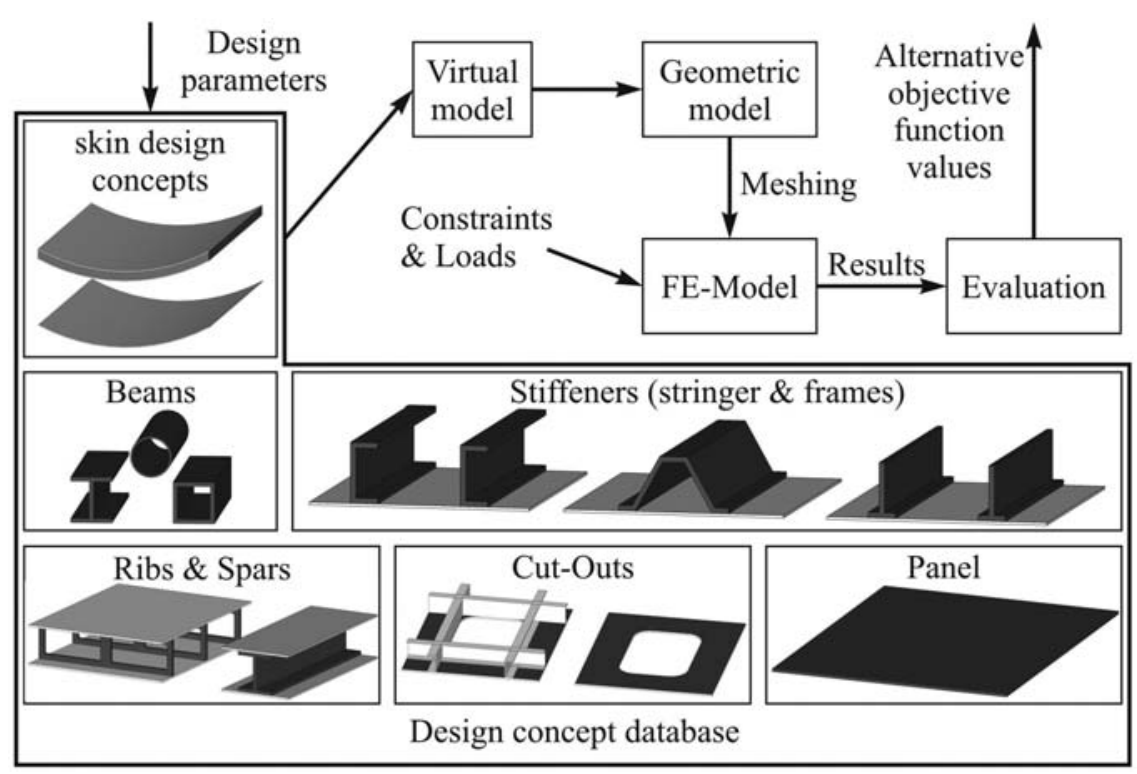

Fig. 6 Flowchart of the simulation model including the design concept database

Based on this information, a virtual model is generated using the database information. This virtual model is needed to check for collisions or other prohibited part dependencies. If such events appear, they will be corrected during this step. Therefore, only feasible structural alternatives are considered when the geometric model is generated. By meshing the geometric model, the finite element model is formed. On this finite element model, all boundary conditions and load cases are applied, followed by the structural simulation. Depending on the objectives, a linear static, buckling, or non-linear analysis of the structure is performed to get the results of stresses, strains, deformations, or eigenvalues. Based on this results, the violations of constraints are calculated to get the penalty factors for this structure. In this last step, the alternative objective function values are calculated as well.

\section{EXAMPLES}

\subsection{Parameterized fuselage model}

The presented design methodology is applied to two aircraft fuselage design problems. Therefore, the general approach of the parameterized simulation model is used to develop a model that creates composite fuselage structures.

The basic structural elements of an aircraft fuselage structure are shown in Fig. 7. The different structural parts implemented in the design concept database will be explained in more detail in the following sections.

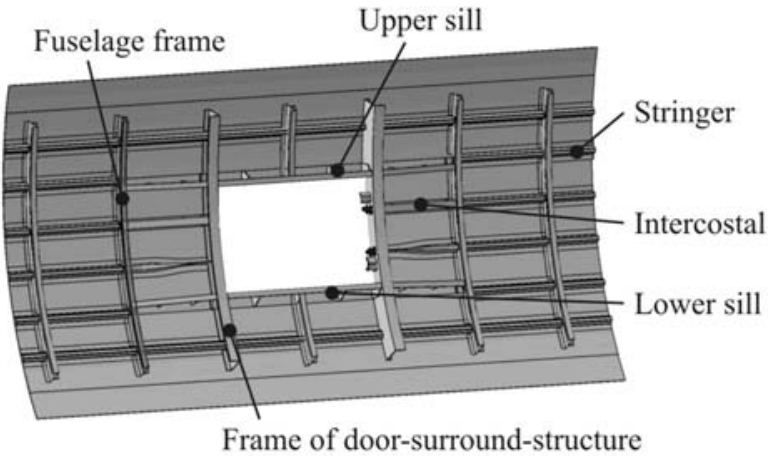

Fig. 7 Structural concept of the parameterized fuselage model

\subsubsection{Skin}

The outer shape of the fuselage skin is defined by several supporting points at different frame stations that are connected by cubic splines. The use of cubic splines allows the parametric model to generate circular as well as double-curved fuselage sections. Also, double-deck configurations, as used in the Airbus A380, can be investigated with this approach. The skin is idealized by eight-node layered shell elements. The design concept implemented so far is a monolithic skin. A sandwich skin architecture is planned but not realized yet.

\subsubsection{Frames}

To stiffen the skin, two different types of frames are implemented in the database. Type 1 is used to stiffen 


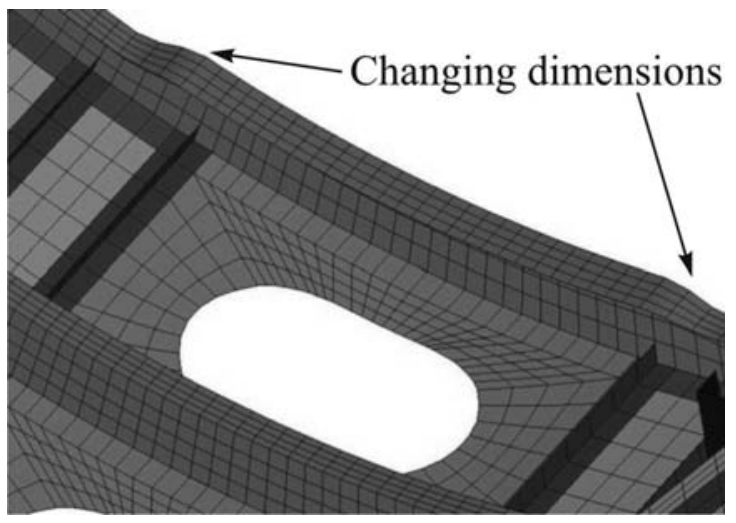

Fig. 8 Changes in frame dimensions

cut-out areas and type 2 is the common frame for fuselage areas without singularities. The frames will be spaced equally on the skin. If cut-outs exist, the cutout frames will be relocated next to the cut-out edges. Both types of frames use separate laminates and the geometry can also be changed independent from each other. They are idealized by eight-node layered shell elements. For the generation of both frame types, four different cross-section layouts (C-, O-, T-, and I-shape) were integrated. The dimensions of the cross-sections can be varied at an arbitrary number of steps at the circumference of the fuselage. This feature allows changes in the height of the webs and the width of the belts, as presented in Fig. 8 .

\subsubsection{Stringers/intercostals/sills}

Other types of stiffeners are the stringers, intercostals, and sills. Seven cross-section shapes are available (C-, O-, Z-, $\Gamma-$, T-, I-, and $\Omega$-shape) for these parts within the database. The sills and intercostals are additional parts and only available if cutouts exist in the structure. The intercostals and sills arise from the stringers but they can have a changed cross-section dimensions at each frame. To consider the different loading conditions at the circumference of airliner fuselages, it is possible to define stringer regions. Within these regions, the number of stringers and the cross-section shape can be varied separately. These stiffeners are also idealized by eight-node layered shell elements.

\subsubsection{Cut-outs}

Cut-outs are no real structural parts but because of the dependencies to other parts, they are also a data set within the database. There are two types of cutouts implemented. The door cut-outs are stiffened by extra layers and a surrounding frame made of sills, door frames, and intercostals. The window cut-outs are stiffened by extra layers only. Depending of the chosen cut-out type, the other structural parts will be arranged.

\subsubsection{Floor structure}

The floor structure consists of several beams and floor plates, as depicted in Fig. 9. It is connected to the surrounding fuselage structure via couplings. The number of crossbeams is equal to the number of frames, whereas the number of seat rails and struts can be varied. Depending on the frame structure, the number of floor plates is fixed. To create floor structures, only the sandwich panel layout and the double-T crosssection shaped beam is implemented in the database.

Summarizing the design variables of all structural parts, a total number of 80 variables and 14 laminates arise. An overview including the data types of the design variables is presented in Table 1.

The diversity of the developed parameterized model is depicted in Fig. 10, where a test panel (a), a double curved fuselage tail section (b), and a fuselage centre section including floor structure (c) is presented.

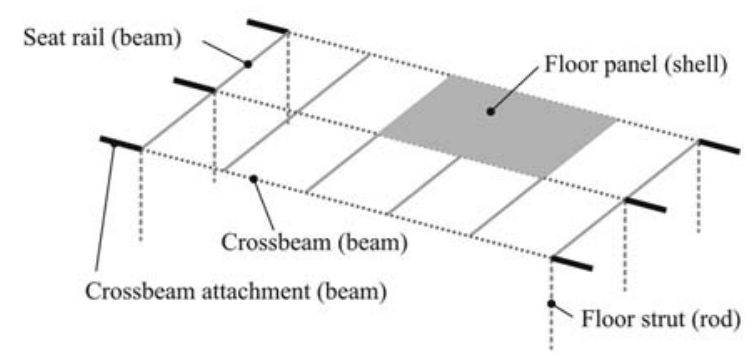

Fig. 9 Schematic of a floor structure

Table 1 Design variables of the fuselage model

\begin{tabular}{ll}
\hline Parameters & Data type \\
\hline $\begin{array}{l}\text { Cross-section shape of fuselage frames, } \\
\text { door frames, intercostals, stringers, }\end{array}$ & Integer \\
upper sills, and lower sills & \\
$\begin{array}{l}\text { Number of fuselage frames, } \\
\text { door frames, stringers, intercostals, }\end{array}$ & Integer \\
upper sills, and lower sills & \\
$\begin{array}{l}\text { Cross-section dimensions of fuselage } \\
\text { frames, door frames, stringers, }\end{array}$ & Real \\
intercostals, upper sills, and lower sills & \\
Width of cut-out stiffening laminates & \\
Distribution of cut-out stiffening laminates & Real \\
Laminate lay-up including angle & Logical \\
and ply material of skin, fuselage frames, & Integer \\
door frames, stringers, intercostals, & \\
upper sills, lower sills, and & \\
cut-out stiffening laminates & \\
\hline
\end{tabular}


(a)

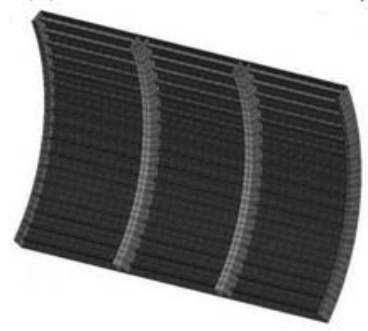

(b)

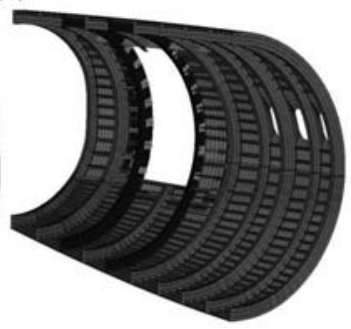

(c)

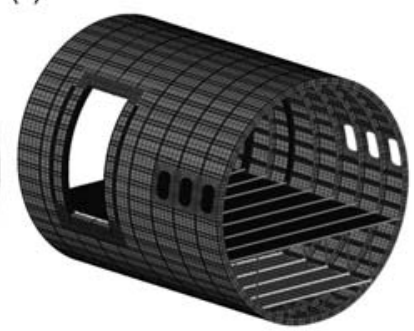

Fig. 10 Structural examples created by the parameterized model

\subsection{Optimization of a composite test panel}

As a first application, this methodology was used to obtain optimal design solutions for a fuselage panel with one cut-out (Fig. 11). This composite test panel (angle $68^{\circ}$, radius $1.98 \mathrm{~m}$, and length $3.5 \mathrm{~m}$ ) has a rectangular cut-out (height $0.6 \mathrm{~m}$ and width $0.9 \mathrm{~m}$ ) and was designed in the EC funded ALCAS project. The depicted design is used as reference for the optimization results.

The objective of the optimization was to find the minimum-weight design with the smallest possible cut-out deformation. Two constraints were considered: no buckling should appear below limit load and no strains should exceed the allowables of the applied materials. The simulation consists of two load cases. Load case 1 is a combination of two loads. A compression force of $356 \mathrm{kN}$ was applied on edge 1 in Fig. 11. Additionally, the structure was loaded by an internal pressure of $0.066 \mathrm{MPa}$. At the opposite edge (edge 2 in Fig. 11), the degrees of freedom of all nodes were fixed except the radial translational direction. At the longitudinal edges (edges 3 and 4 in Fig. 11), symmetry boundary conditions were applied. The second load case contains the same boundary conditions and the same internal pressure. However, instead of the compression force, a torsional load of $176.5 \mathrm{kN}$ was applied.

Results obtained were the mass of the structure, strains in the laminates, and the deformation of the

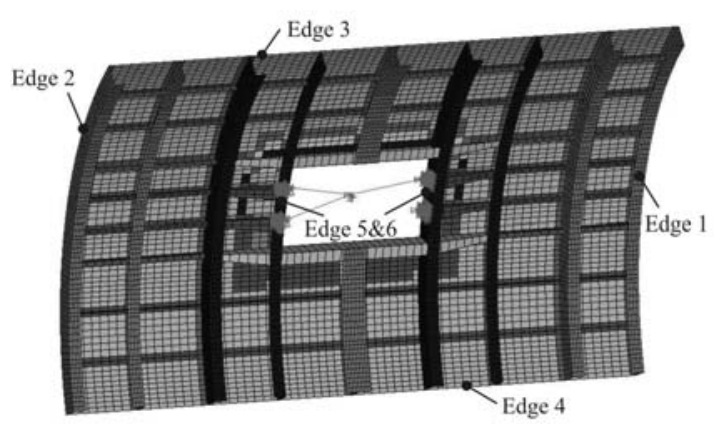

Fig. 11 Reference composite test panel cut-out, which was computed by the maximum relative deformation between the opposing nodes on edges 5 and 6 in Fig. 11. Based on these values, the penalty factor and the values of the alternative objective functions were calculated.

The optimization was performed with populations consisting of 70 individuals. In every 20th generation, the optimization method switched between ESs/GAs and the local search with DE. After 100 generations, the design space was adjusted. The optimization was stopped after 200 generations. Former test optimizations showed a good compromise between the run time and the convergence of the results for this number of generations. In Fig. 12, the best individuals of the population with their mass and cut-out deformation are compared to the reference panel.

The results of four panels highlighted in Fig. 12 are summarized in Table 2. Panel 1 is the design with the lowest structural mass, whereas Panel 4 has the lowest cut-out deformation. However, more

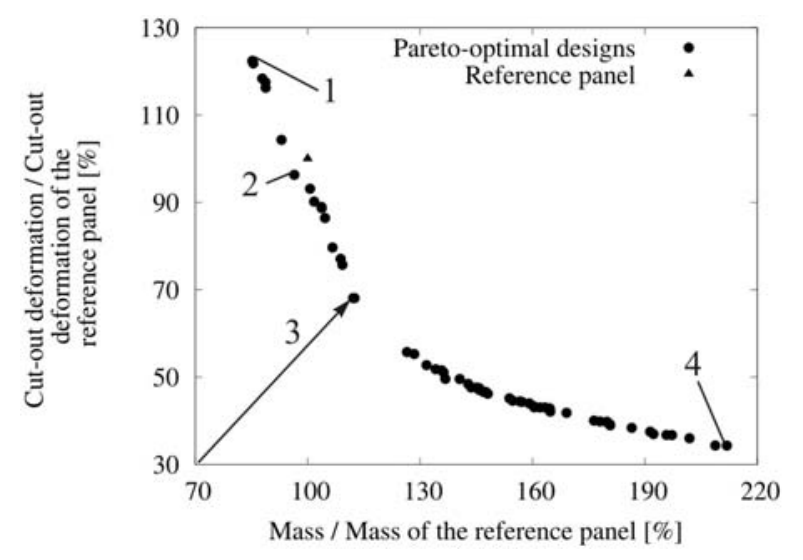

Fig. 12 Pareto front of the test panel optimization

Table 2 Results of the test panel optimization

\begin{tabular}{lllll}
\hline & \multicolumn{4}{c}{ Panel } \\
\cline { 2 - 5 } & 1 & 2 & 3 & 4 \\
\hline Mass/mass of reference panel (\%) & 85.24 & 96.43 & 112.13 & 211.86 \\
$\begin{array}{l}\text { Cut-out deformation/cut-out } \\
\text { deformation of reference panel (\%) }\end{array}$ & 122.39 & 96.31 & 68.08 & 34.32 \\
\hline
\end{tabular}



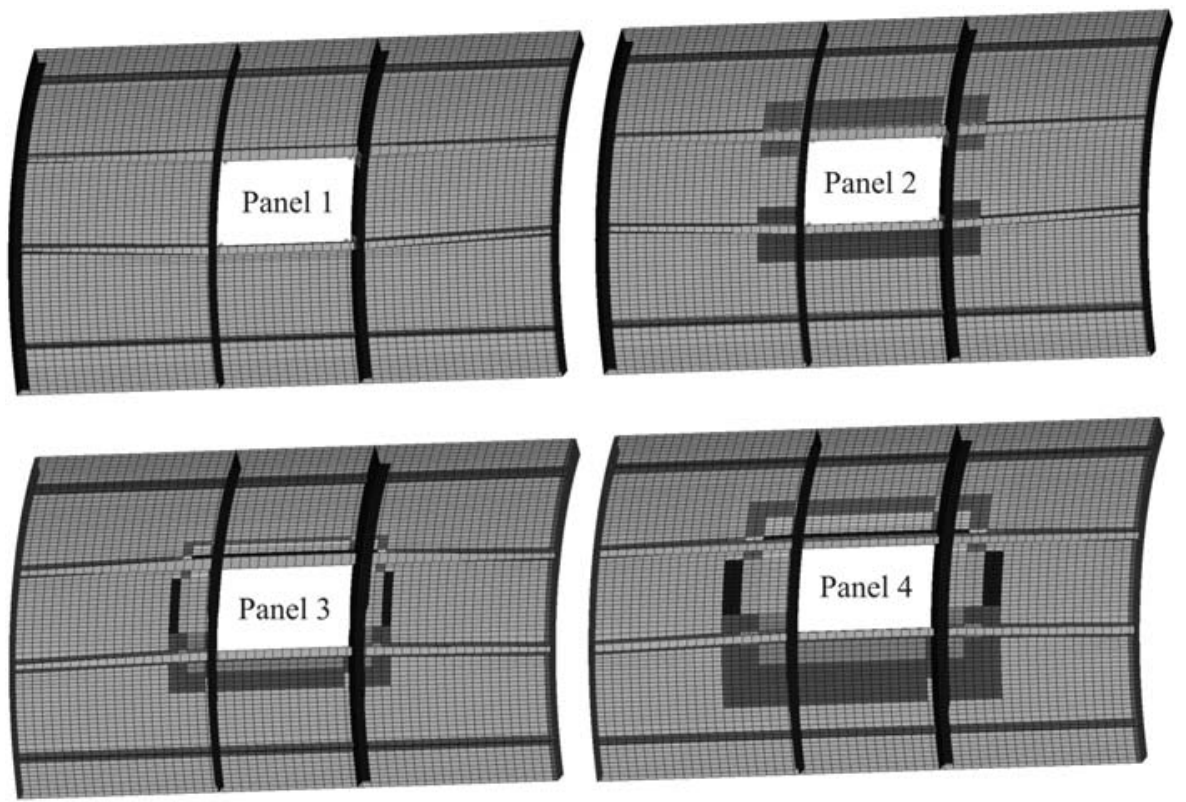

Fig. 13 Selected structural examples of the test panel optimization

interesting is Panel 2. It has a lower structural mass as well as a smaller cut-out deformation. Hence, it is an improvement in both objectives compared to the reference panel. Panel 3 is the best compromise solution with the smallest position vector in the alternative objective function plane. It has a higher mass but a significantly lower cut-out deformation. The designs of all four panels are shown in Fig. 13.

\subsection{Optimization of a composite fuselage section}

Within a second application, the presented design methodology was used to optimize a 4.8 -m long composite tail section of a single-aisle airliner fuselage (Fig. 14). The barrel has a passenger door (height $2.1 \mathrm{~m}$ and width $1.0 \mathrm{~m}$ ) and three window cut-outs (height $0.41 \mathrm{~m}$ and width $0.3 \mathrm{~m}$ ) on each side. Due to the symmetry, only one half of the structure was used in the finite element analysis.

The aim of this optimization was also to find the minimum-weight design with the smallest possible cut-out deformation. The basic constraints considered in this design problem were that the strain allowables of the applied materials should not be exceeded. To reduce the calculation time, no linear buckling analysis was performed in this optimization run.

Two load cases were applied to the structure. The first one was a combination of an axial compression force of $931 \mathrm{kN}$ and an internal pressure of $0.066 \mathrm{MPa}$. The compression load was applied on edge 1 in Fig. 14. The second load case consisted of the same internal pressure but a torsional load of $465 \mathrm{kN}$ that was also applied to edge 1 in Fig. 14. At the opposite edge (edge 2 in Fig. 14), all nodes were constrained for both load cases. At these nodes, all degrees of freedom were fixed except the radial translational direction. At the longitudinal edges (edges 3 and 4 in Fig. 14), symmetry boundary conditions were applied.

Results obtained were the mass of the structure, strains in the laminates, and the deformation of the door cut-out, which was computed by the maximum relative deformation between the opposing nodes on edges 5 and 6 in Fig. 14. Based on these values, the penalty factor and the values of the alternative objective functions were calculated.

The structure depicted in Fig. 14 was designed by conventional design methods and is able to

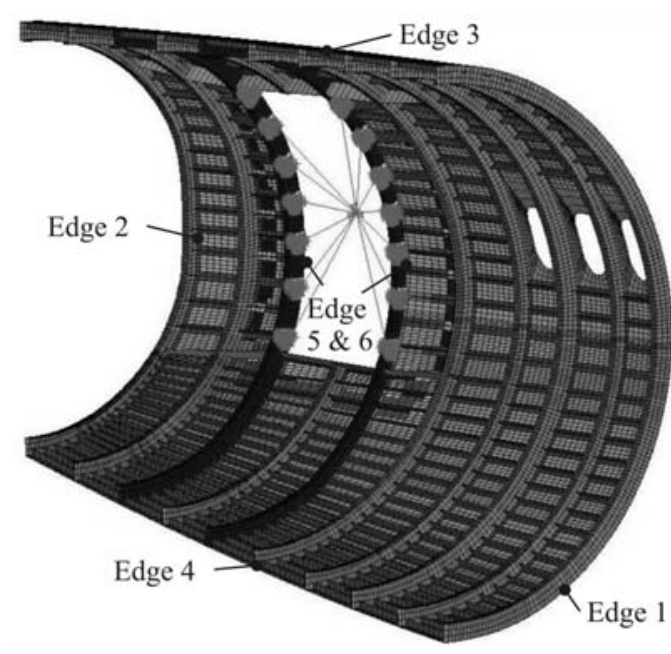

Fig. 14 Reference fuselage tail section 
Table 3 Results of the fuselage tail section optimization

\begin{tabular}{|c|c|c|c|c|}
\hline & \multicolumn{4}{|c|}{ Structure } \\
\hline & 1 & 2 & 3 & 4 \\
\hline $\begin{array}{l}\text { Mass/mass of reference } \\
\text { structure (\%) }\end{array}$ & 77.12 & 79.03 & 96.64 & 170.78 \\
\hline $\begin{array}{l}\text { Cut-out deformation/cut-out } \\
\text { deformation of reference } \\
\text { structure }(\%)\end{array}$ & 110.06 & 98.93 & 73.34 & 42.15 \\
\hline
\end{tabular}

carry both load cases without violating the given constraints. This design solution was used as a reference for the evaluation of this optimization results.

The optimization was performed with populations consisting of 60 individuals. In every 30 th generation, the optimization method switched between ESs/GAs and the local search with DE. This optimization was stopped after 200 generations as well. In Fig. 15, the best individuals of the population with their mass and cut-out deformation are compared to the reference structure.

The results of four structures highlighted in Fig. 15 are summarized in Table 3. Structure 1 is the design with the lowest structural mass, whereas Structure 4 has the lowest cut-out deformation. The Structures 2 and 3 offer improvements in one objective compared to the reference structure. Structure 2 has a similar

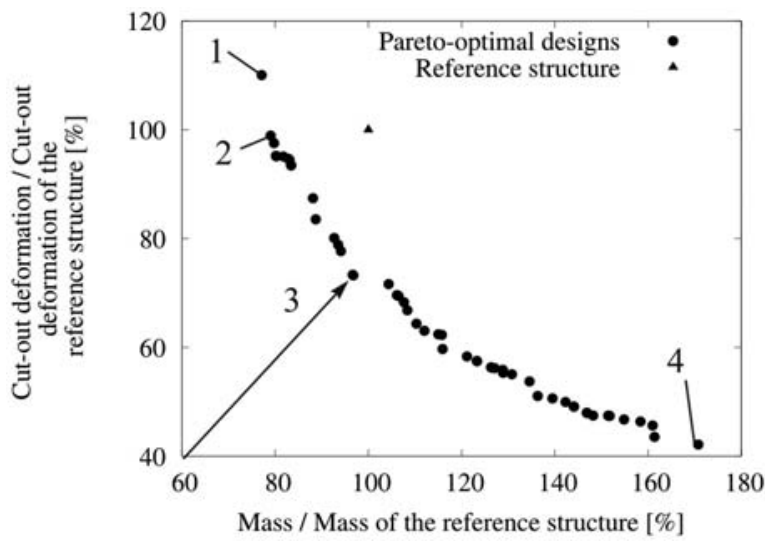

Fig. 15 Pareto front of the fuselage tail section optimization

cut-out deformation but a significant lower mass, while Structure 3 has nearly the same mass but a lower cut-out deformation. The latter is also the best compromise solution. The designs of the four highlighted structures are depicted in Fig. 16.

\section{SUMMARY}

A design methodology based on EAs that is applicable to complex composite aircraft structures was presented. The challenging task of designing

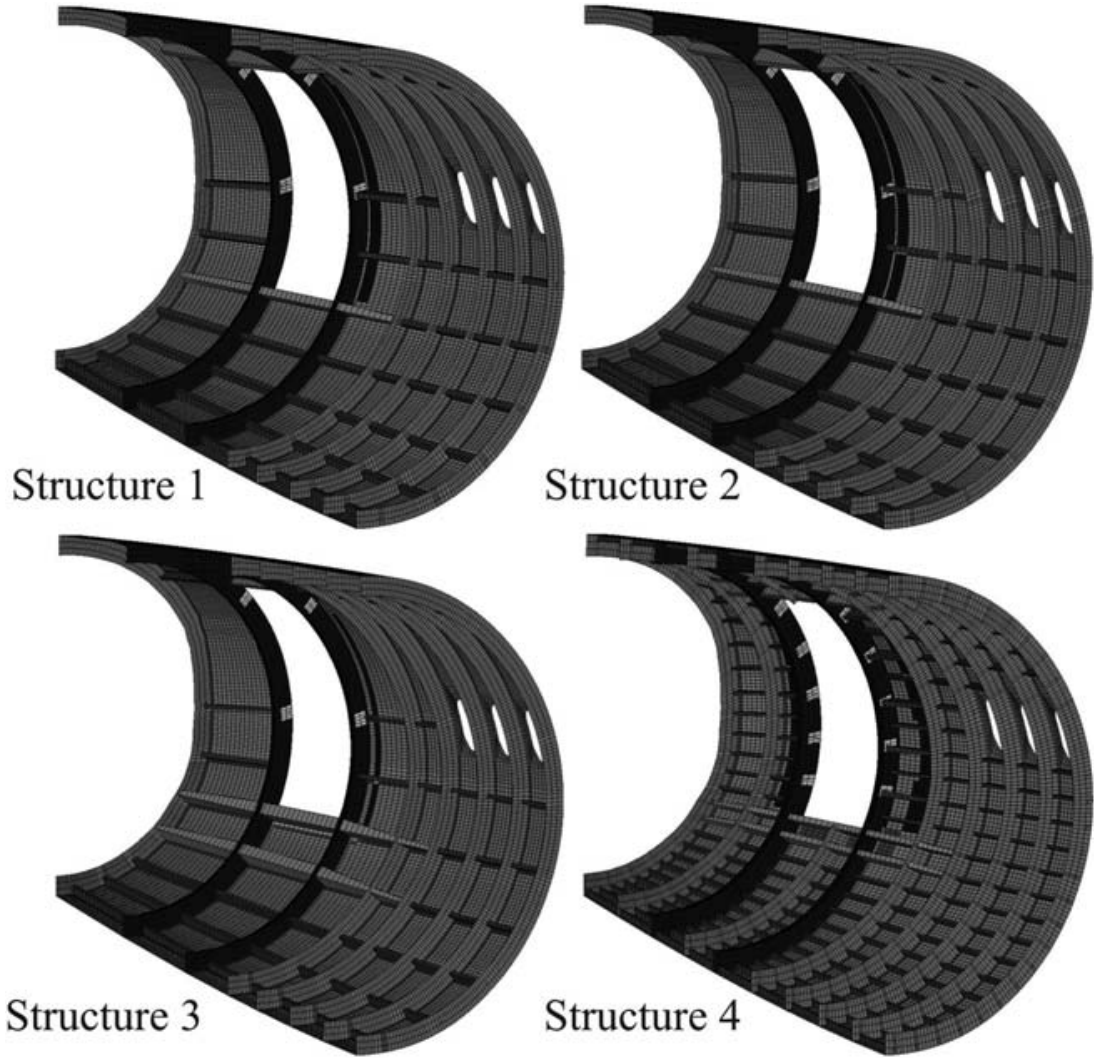

Fig. 16 Selected structural examples of the fuselage tail section optimization 
low-weight structures considering conflicting objectives simultaneously can be solved efficiently using this approach. The applicability of the developed tool was proved by designing a CFRP test panel as well as a composite tail section of a single-aisle airliner fuselage including cut-outs.

The presented design concept database approach within a parameterized finite element model allows an easy integration of further skin design concepts as well as additional stiffener layouts or other parts. Furthermore, the used multi-objective approach offers the opportunity to integrate manufacturing aspects as well. Therefore, this methodology offers the possibility to consider interdisciplinary aspects in the design process as well.

\section{FUNDING}

This study was partially funded by the 7th Framework Program of the European Union during the ALCAS project (AIP4-CT-2003-516092). The financial support provided by the European Union is gratefully acknowledged.

(C) TU Dresden/Institute of Aerospace Engineering 2011

\section{REFERENCES}

1 Conceição António, C. A. A multilevel genetic algorithm for optimization of geometrically nonlinear stiffened composite structures. Struct. Multidisciplin. Optim., 2002, 24, 372-376.

2 Gallet, C., Salaüin, M., and Bouchet, E. An example of global structural optimization with genetic algorithms in the aerospace field. In Proceedings of the 8th International Conference on Computational plasticity, 5-8 September 2005, Barcelona, Spain.

3 Conceição António, C. A. A hierarchical genetic algorithm with age structure for multimodal optimal design of hybrid composites. Struct. Multidisciplin. Optim., 2006, 31, 280-294.

4 Faggiani, A. and Falzon, B. G. Optimization strategy for minimizing damage in postbuckling stiffened panels. AIAA J., 2007, 45, 2520-2528.

5 Iuspa, L. and Ruocco, E. Optimum topological design of simply supported composite stiffened panels via genetic algorithms. Comput. Struct., 2008, 86, 1718-1737.

6 Gantovnik, V. B., Gürdal, Z., and Watson, L. T. A genetic algorithm with memory for optimal design of laminated sandwich composite panels. In Proceedings of the 43rd AIAA/ASME/ASCE/AHS/ ASC Structures, structural dynamics and materials conference, 22-25 April 2002, Denver, Colorado, AIAA Paper No. 2002-1221.

7 Liu, B., Haftka, R. T., Akgün, M. A., and Todoroki, A. Permutation genetic algorithm for stacking sequence design of composite laminates. Comput. Meth. Appl. Mech. Eng., 2000, 186, 357-372.

8 Soremekun, G., Gürdal, Z., Haftka, R. T., and Watson, L. T. Composite laminate design optimization by genetic algorithm with generalized elitist selection. Comput. Struct, 2001, 79, 131-143.

9 Kaletta, P. and Wolf, K. Optimization of composite aircraft panels using evolutionary computation methods. In Proceedings of the 22nd ICAS Conference, Harrogate, UK, 28 August-1 September 2000, pp. 411.1-411.10.

10 Spallino, R. Structural optimization of a CFRP fuselage section by differential evolution. In Proceedings of the 15th AIMETA Congress of Theoretical and Applied Mechanics, Taormina, Italy, 26-29 September 2001.

11 Wolf, K. Optimization of composite sandwich panels using evolutionary computation methods. In Proceedings of the 42nd AIAA/ASME/ASCE/AHS Structures, structural dynamics and materials conference, 16-19 April, 2001, Seattle, Washington, AIAA Paper 2001-1277, pp. 1-11.

12 Keller, D. Optimization of ply angles in laminated composite structures by a hybrid, asynchronous, parallel evolutionary algorithm. Compos. Struct., 2010, 92, 2781-2790.

13 Spallino, R. and Rizzo, S. Multi-objective discrete optimization of laminated structures. Mech. Res. Commun., 2002, 29, 17-25.

14 Irisarri, F. X., Hicham Basir, D., Carrere, N., and Maire, J. F. Multiobjective stacking sequence optimization for laminated composite structures. Compos. Sci. Technol., 2009, 69, 983-990.

15 Seeger, J. and Wolf, K. Structural optimization of adaptive airfoils using evolutionary algorithms. In Proceedings of the 1st CEAS European Air and Space Conference, 10-13 September 2007, Berlin, Germany.

16 Haftka, R. T. and Gürdal, Z. Elements of structural optimization, third edition, 1991 (Kluwer Academic Publishers, Dordrecht, Boston, London).

17 Osyczka, A. Evolutionary algorithms for single and multicriteria design optimization, 2002 (PhysicaVerlag, Heidelberg, New York).

18 Marler, R. T. and Arora, J. S. Survey of multi-objective optimization methods for engineering. Struct. Multidisciplin. Optim., 2004, 26, 369-395.

19 Kaletta, P. Ein Beitrag zur Effizienzsteigerung Evolutionärer Algorithmen zur optimalen Auslegung von Faserverbundstrukturen im Flugzeugbau. PhD thesis, 2006.

20 Bäck, T., Hoffmeister, F., and Schwefel, H. P. A survey of evolution strategies. In Proceedings of the 4th International Conference on Genetic Algorithms, San Diego, California, (Morgan Kaufman). 1991, pp. 2-9.

21 Storn, R. and Price, K. Differential evolution - a simple and efficient heuristic for global optimization over continuous spaces. J. Global Optim., 1997, 11, 341-359.

22 Zhang, Q., Liu, W., and Li, H. The performance of a new version of MOEA/D on CEC2009 unconstrained 
MOP test instances. In Proceedings of the CEC2009, Trondheim, 18-21 May 2009, pp. 203-208.

23 Zhang, J. and Sanderson, A. C. JADE: Adaptive differential evolution with optional external archive. IEEE Trans. Evol. Comput., 2009, 13, 945-958.

24 Wang, W., Guo, S., Chang, N., and Yang, W. Optimum buckling design of composite stiffened panels using ant colony algorithm. Compos. Struct., 2010, 92, 712-719.

25 Yang, B. Modified particle swarm optimizers and their application to robust design and structural optimization. PhD thesis, 2009.

26 Zhang, Q., Zhou, A., Zhou, S., Suganthan, P. N., Liu, W., and Tiwari, S. Multiobjective optimization test instances for the CEC 2009 special session and competition. Technical Report for the CEC2009, 2009.

27 Tang, K., Li, X., Suganthan, P. N., Yang, Z., and Weise, T. Benchmark functions for the CEC'2010 special session and competition on large-scale global optimization. Technical Report for the CEC2010, 2010.

\section{APPENDIX}

\section{Notation}

$f \quad$ objective function

$f^{E} \quad$ alternative objective function

$\boldsymbol{f}^{\boldsymbol{E}} \quad$ vector of alternative objective functions

g vector of inequality constraints

$\boldsymbol{h}$ vector of equality constraints

$n \quad$ number of design variables

$P \quad$ penalty term

$q$ number of alternative objective functions

$\boldsymbol{x}$ design vector

$x_{i} \quad$ design variable

$\Omega \quad$ design space

$\Omega_{\mathrm{f}} \quad$ feasible region of the design space

$\Omega_{\text {inf }}$ infeasible region of the design space. 